\title{
THE $\Theta$-SPHERICAL TRANSFORM AND ITS INVERSION
}

\author{
ANGELA PASQUALE
}

\begin{abstract}
The $\Theta$-spherical transform is defined as a simultaneous generalization of the Harish-Chandra's spherical transform on Riemannian symmetric spaces of noncompact type and of the spherical Laplace transform on noncompactly causal symmetric spaces as defined by Faraut, Hilgert and Ólafsson. An extension of Ólafsson's expansion formula allows us to deduce an inversion formula for the $\Theta$-spherical transform on the space of $W_{\Theta}$-invariant $C^{\infty}$ functions with compact support.
\end{abstract}

\section{Introduction}

Harish-Chandra's theory of spherical functions on Riemannian symmetric spaces of noncompact type has been recently generalized in two different directions. On one side, there has been a sort of analytic continuation of HarishChandra's theory to a, in general non-geometric, context in which the spherical functions are considered as depending on additional complex parameters (the multiplicities). This is the theory of hypergeometric functions associated with root systems, constructed by Heckman and Opdam from the late 1980s (see e.g. [5], [6], [17] and references therein). Their construction was motivated by the theory of special functions. In one variable, the spherical functions on Riemannian symmetric spaces of rank-one are special cases of the Gaussian hypergeometric functions (or, more precisely, of the Jacobi functions of the first kind). Heckman and Opdam moved into the opposite direction and constructed hypergeometric functions in more variables by analytically continuing in the multiplicities the spherical functions on Riemannian symmetric spaces of higher rank. On the other side, there have been attempts of extending HarishChandra's theory to non-Riemannian symmetric spaces. A class in which the extension turned out to be quite successful consists of the so-called noncompactly causal (NCC) symmetric spaces. The theory of spherical functions on these spaces started in the 1990s with the work of Faraut, Hilgert and Ólafsson (see e.g. [1], [8] and [10]).

In [18] we have developed a theory of spherical functions which generalizes Heckman-Opdam's theory of hypergeometric functions associated with

Received August 2, 2002; in revised form March 10, 2003. 
root systems and which also includes as special geometric instance the theory of Faraut-Hilgert-Ólafsson (see also [11]). These generalized functions have been called $\Theta$-spherical functions because, for a fixed geometric multiplicity function on a root system $\Sigma$, the different geometric situations (e.g. Riemannian or NCC) are obtained by selecting a set $\Theta$ of positive simple roots in $\Sigma$.

In this paper we define and study the integral transform associated with the $\Theta$-spherical functions, which we call the $\Theta$-spherical transform. By construction it contains as special cases Opdam's transform [16], hence HarishChandra's spherical transform, and the spherical Laplace transform as defined in [1].

Suppose $\Sigma$ is a root system in the dual space $\mathfrak{a}^{*}$ of a Euclidean space $\mathfrak{a}$. For a fixed multiplicity function $m$ on $\Sigma$, each $\Theta$-spherical function $\varphi_{\Theta}(m ; \lambda, a)$ depends on two variables: a spectral parameter $\lambda$ in the complex dual $a_{c}^{*}$ of $a$, and a space parameter $a$ in a certain open domain $A_{\Theta}$ in a split Cartan space $A$ with Lie algebra $a$. More precisely, $A_{\Theta}=\exp a_{\Theta}$, where $\mathfrak{a}_{\Theta}$ is an open convex cone containing the positive Weyl chamber $\mathfrak{a}^{+}$and invariant under the Weyl group $W_{\Theta}$ of the root system generated by $\Theta$. Let $f$ be a sufficiently regular $W_{\Theta}$-invariant function on $A_{\Theta}$. The $\Theta$-spherical Fourier transform of $f$ is the $W_{\Theta}$-invariant function $\mathscr{F}_{\Theta} f(m)$ on $a_{C}^{*}$ defined by

$$
\mathscr{F}_{\Theta} f(m ; \lambda):=\frac{1}{\left|W_{\Theta}\right|} \int_{A_{\Theta}} f(a) \varphi_{\Theta}(m ; \lambda, a) \delta(m ; a) d a,
$$

where $\delta(m ; a)$ is a certain density and $d a$ is a suitable normalization of the Haar measure on $A$. We refer to Sections 2 and 3 for the precise definitions.

Our main result is the inversion of the $\Theta$-spherical transform on the space of $W_{\Theta}$-invariant $C^{\infty}$ functions with compact support in $A_{\Theta}$. The resulting inversion formula extends the formula of Ólafsson for the inversion of the spherical Laplace transform on NCC symmetric spaces [10]. In the case of NCC spaces, the inversion has been obtained by reduction to the Riemannian case. We follow the same approach here and reduce the case of the $\Theta$-spherical transform to the inversion of Opdam's transform [16]. The proof has two main ingredients: (a) An easy generalization of Ólafsson's functional relation, which allows us to write the the hypergeometric functions associated with root systems as linear combinations of arbitrary $\Theta$-spherical functions; (b) Various estimates for the $\Theta$-spherical functions developed in [18], which guarantee the convergence of the integrals involved in the computations.

ACKNOWLEDGEMENTS. This paper is part of the Habilitationsschrift of the author. She wishes to express her gratitude to Joachim Hilgert, Gestur Ólafsson and Eric Opdam for valuable discussions. 


\section{Notation and preliminaries}

Let $a$ be an $l$-dimensional real Euclidean vector space with inner product $\langle\cdot, \cdot\rangle$. For every $\lambda$ in the dual space $\mathfrak{a}^{*}$ of $\mathfrak{a}$, let $A_{\lambda} \in \mathfrak{a}$ be determined by the condition that $\lambda(H)=\left\langle H, A_{\lambda}\right\rangle$ for all $H \in \mathfrak{a}$. If $\lambda \neq 0$, then $H_{\lambda}:=2 A_{\lambda} /\left\langle A_{\lambda}, A_{\lambda}\right\rangle$ satisfies $\lambda\left(H_{\lambda}\right)=2$. The assignment $\langle\lambda, \mu\rangle:=\left\langle A_{\lambda}, A_{\mu}\right\rangle$ defines an inner product in $\mathfrak{a}^{*}$. The complexification $\mathfrak{a}_{\mathrm{C}}:=\mathfrak{a} \otimes_{\mathrm{R}} \mathrm{C}$ of $\mathfrak{a}$ can be viewed as the Lie algebra of the complex torus $A_{\mathrm{C}}:=\mathfrak{a}_{\mathrm{C}} / \mathrm{Z}\left\{i \pi H_{\alpha}: \alpha \in \Sigma\right\}$. We write $\exp : a_{C} \rightarrow A_{C}$ for the exponential map, with multi-valued inverse log. The split real form $A:=\exp \mathfrak{a}$ of $A_{\mathrm{C}}$ is an abelian subgroup of $A_{\mathrm{C}}$ with Lie algebra a such that exp : $\mathfrak{a} \rightarrow A$ is a diffeomorphism. The polar decomposition of $A_{\mathcal{C}}$ is $A_{\mathrm{C}}=A T$, where $T:=\exp (i a)$ is a compact torus with Lie algebra $i$ a.

Let $\Sigma$ be a (not necessarily reduced) root system in the dual $\mathfrak{a}^{*}$ with associated Weyl group $W$. For every $\alpha \in \Sigma$, we denote by $r_{\alpha}$ the reflection in $\mathfrak{a}^{*}$ defined by $r_{\alpha}(\lambda):=\lambda-\lambda\left(H_{\alpha}\right) \alpha$ for all $\lambda \in \mathfrak{a}^{*}$. Let $\Sigma^{+}$be a choice of positive roots in $\Sigma$ and $\Pi=\left\{\alpha_{1}, \ldots, \alpha_{l}\right\}$ the fundamental system of simple roots associated with $\Sigma^{+}$. We respectively denote by $\Sigma_{\mathrm{i}}^{+}$and $\Sigma_{\mathrm{u}}^{+}$the indivisible and unmultipliable roots in $\Sigma^{+}$. The positive Weyl chamber $\mathfrak{a}^{+}$consists of the elements $H \in \mathfrak{a}$ for which $\alpha(H)>0$ for all $\alpha \in \Sigma^{+}$. We set $A^{+}:=\exp \mathfrak{a}^{+}$.

Let $a_{\mathrm{C}}^{*}$ be the space of all C-linear functionals on $a$. The C-bilinear extension to $\mathfrak{a}_{\mathrm{C}}^{*}$ and $\mathfrak{a}_{\mathrm{C}}$ of the inner products $\langle\cdot, \cdot\rangle$ on $\mathfrak{a}^{*}$ and $\mathfrak{a}$ will also be denoted by $\langle\cdot, \cdot\rangle$. The action of $W$ extends to $a$ by duality, to $a_{C}^{*}$ and $a_{C}$ by C-linearity, and to $A_{\mathrm{C}}$ and $A$ by the exponential map. Moreover, $W$ acts on functions $f$ on any of these spaces by $(w f)(x):=f\left(w^{-1} x\right), w \in W$.

A multiplicity function on $\Sigma$ is a $W$-invariant function $m: \Sigma \rightarrow C$. Setting $m_{\alpha}:=m(\alpha)$ for $\alpha \in \Sigma$, we therefore have $m_{w \alpha}=m_{\alpha}$ for all $w \in W$. The set $\mathcal{M}$ of all multiplicity functions on $\Sigma$ is a subspace of the finite-dimensional C-vector space $\mathrm{C}^{\Sigma}$. The subset of $\mathscr{M}$ consisting of all multiplicity functions $m$ with $m_{\alpha} \geq 0$ for all $\alpha \in \Sigma$ is denoted by $\mathscr{M}^{+}$. A multiplicity function $m \in \mathscr{M}^{+}$ is said to be even if $m_{\alpha} \in 2 \mathrm{~N}$ for all $\alpha \in \Sigma$. We say that $m$ is geometric if there is a Riemannian symmetric space of noncompact type $G / K$ with restricted root system $\Sigma$ such that $m_{\alpha}$ is the multiplicity of the root $\alpha$ for all $\alpha \in \Sigma$. Otherwise, $m$ is said to be non-geometric.

We adopt the multiplicity notation commonly used in the theory of symmetric spaces. It differs from the notation employed by Heckman and Opdam in the following ways. The root system $R$ used by Heckman and Opdam is related to our root system $\Sigma$ by the relation $R=\{2 \alpha: \alpha \in \Sigma\}$; the multiplicity function $k$ in Heckman-Opdam's work is related to our $m$ by $k_{2 \alpha}=m_{\alpha} / 2$.

For $\alpha \in \Sigma$ and $\lambda \in \mathfrak{a}_{\mathrm{C}}^{*}$ we set

$$
\lambda_{\alpha}:=\frac{\lambda\left(H_{\alpha}\right)}{2}=\frac{\langle\lambda, \alpha\rangle}{\langle\alpha, \alpha\rangle} .
$$


The restricted weight lattice of $\Sigma$ is the set $P$ of all $\lambda \in \mathfrak{a}^{*}$ with $\lambda_{\alpha} \in \mathrm{Z}$ for all $\alpha \in \Sigma$. Notice that $2 \alpha \in P$ for all $\alpha \in \Sigma$. The elements of $a_{\mathrm{C}}^{*} \backslash P$ are said to be generic.

If $\lambda \in P$, then the exponential $e^{\lambda}$ defined by $e^{\lambda}(a):=e^{\lambda(\log a)}$ is single valued on $A_{\mathrm{C}}$. The $\mathrm{C}$-linear span of the $e^{\lambda}$ is the ring $\mathrm{C}\left[A_{\mathrm{C}}\right]$ of regular functions on the affine algebraic variety $A_{\mathrm{C}}$. The lattice $P$ is $W$-invariant, and the Weyl group acts on $\mathrm{C}\left[A_{\mathrm{C}}\right]$ according to $w\left(e^{\lambda}\right):=e^{w \lambda}$. The algebra $\mathrm{C}\left[A_{\mathrm{C}}^{\text {reg }}\right]$ of regular functions on $A_{\mathrm{C}}^{\mathrm{reg}}:=\left\{h \in A_{\mathrm{C}}: e^{2 \alpha(\log h)} \neq 1\right.$ for all $\left.\alpha \in \Sigma\right\}$ is the subalgebra of the quotient field $\mathrm{C}\left(A_{\mathrm{C}}\right)$ of $\mathrm{C}\left[A_{\mathrm{C}}\right]$ generated by $\mathrm{C}\left[A_{\mathrm{C}}\right]$ and by $1 /\left(1-e^{-2 \alpha}\right)$ for $\alpha \in \Sigma^{+}$. Its $W$-invariant elements form the subalgebra $\mathrm{C}\left[A_{\mathrm{C}}^{\text {reg }}\right]^{W}$. Notice that $A^{+} \subset A_{\mathrm{C}}^{\mathrm{reg}}$.

Let $\mathrm{S}\left(\mathfrak{a}_{\mathrm{C}}\right)$ denote the symmetric algebra over $\mathfrak{a}_{\mathcal{C}}$ considered as the space of polynomial functions on $\mathfrak{a}_{\mathrm{C}}^{*}$, and let $\mathrm{S}\left(\mathfrak{a}_{\mathrm{C}}\right)^{W}$ be the subalgebra of $W$-invariant elements. We shall also indicate with $\mathrm{S}\left(\mathfrak{a}_{\mathrm{C}}\right)$ the algebra of the corresponding constant-coefficient differential operators $\partial(p)$ on $A_{C}$ and $a_{c}$. The algebra of differential operators on $A_{\mathrm{C}}$ with coefficients in $\mathrm{C}\left[A_{\mathrm{C}}^{\mathrm{reg}}\right]$ is denoted by $\mathrm{D}\left(A_{\mathrm{C}}^{\mathrm{reg}}\right):=\mathrm{C}\left[A_{\mathrm{C}}^{\mathrm{reg}}\right] \otimes \mathrm{S}\left(\mathfrak{a}_{\mathrm{C}}\right)$. The Weyl group $W$ acts on $\mathrm{D}\left(A_{\mathrm{C}}^{\text {reg }}\right)$ according to

$$
w(\varphi \otimes \partial(p)):=w \varphi \otimes \partial(w p) .
$$

We write $\mathrm{D}\left(A_{\mathrm{C}}^{\mathrm{reg}}\right)^{W}$ for the subspace of $W$-invariant elements. Considering $D \in \mathrm{D}\left(A_{\mathrm{C}}^{\mathrm{reg}}\right)$ as element of $\mathrm{D}\left(A_{\mathrm{C}}^{\mathrm{reg}}\right) \otimes \mathrm{C}[W]$, we shall usually write $D$ instead of $D \otimes 1$.

Let $L_{A}$ denote the Laplace operator on $A$. Then

$$
L(m):=L_{A}+\sum_{\alpha \in \Sigma^{+}} m_{\alpha} \frac{1+e^{-2 \alpha}}{1-e^{-2 \alpha}} \partial\left(A_{\alpha}\right)
$$

generalizes to arbitrary multiplicity functions $m$ the radial component on $A^{+}$of the Laplace operator on a Riemannian symmetric space $G / K$ of noncompact type. Heckman and Opdam proved in [5] that the commutant $\mathrm{D}(\mathfrak{a}, m, \Sigma):=$ $\left\{Q \in \mathrm{D}\left(A_{\mathrm{C}}^{\mathrm{reg}}\right)^{W}: L(m) Q=Q L(m)\right\}$ of $L(m)$ in $\mathrm{D}\left(A_{\mathrm{C}}^{\mathrm{reg}}\right)^{W}$ is a commutative algebra which plays the role for the triple $(\mathfrak{a}, \Sigma, m)$ of the commutative algebra of the radial components on $A^{+}$of the invariant differential operators on a Riemannian symmetric space of noncompact type. The algebra $\mathrm{D}(\mathfrak{a}, m, \Sigma)$ can be constructed algebraically. Indeed one has

$$
\mathrm{D}(\mathfrak{a}, m, \Sigma):=\left\{D(p, m): p \in \mathrm{S}\left(\mathfrak{a}_{\mathrm{C}}\right)^{W}\right\},
$$

where the differential operator $D(p, m)$ can be explicitly given in terms of Dunkl-Cherednik operators (see [4]). For instance, one has

$$
L(m)+\langle\rho(m), \rho(m)\rangle=D\left(p_{L}, m\right),
$$


where $p_{L}(\lambda)=\langle\lambda, \lambda\rangle$ and

$$
\rho(m):=\frac{1}{2} \sum_{\alpha \in \Sigma^{+}} m_{\alpha} \alpha
$$

Let $\lambda \in a_{C}^{*}$ be fixed. The system of differential equations

$$
D(p, m) \varphi=p(\lambda) \varphi, \quad p \in \mathrm{S}\left(a_{\mathrm{C}}\right)^{W},
$$

is called the hypergeometric system of differential equations with spectral parameter $\lambda$ associated with the data $(a, \Sigma, m)$. For geometric multiplicities, the hypergeometric system (4) agrees with the system of differential equations on $A$ defining Harish-Chandra's spherical function of spectral parameter $\lambda$.

In the following we shall consider meromorphic functions $f$ on $a_{C}^{*}$ with singular locus contained in the union of a locally finite (generally infinite) family $\mathbf{H}$ of affine complex hyperplanes $\mathscr{H}_{r, \alpha}=\left\{\lambda \in a_{c}^{*}: \lambda_{\alpha}=r\right\}$. We say that $f$ has at most a simple pole along the hyperplane $\mathscr{H}_{r, \alpha}$ if the function $\lambda \mapsto$ $\left(\lambda_{\alpha}-r\right) f(\lambda)$ extends to be holomorphic in a neighborhood of $\mathscr{H}_{r, \alpha} \backslash \bigcup_{\mathscr{H} \in \mathbf{H}} \mathscr{H}$.

\section{2. $\Theta$-spherical functions}

At every point $h \in A_{\mathrm{C}}^{\mathrm{reg}}$, the space of local solutions of (4) near $h$ consists of holomorphic functions and its dimension is the order $|W|$ of the Weyl group, that is the hypergeometric system is holonomic of rank $|W|$ (cf. [6], Corollary 4.1.8. See also [17], Theorem 6.7). Paralleling Harish-Chandra's work, Heckman and Opdam [5] constructed for generic $\lambda \in \mathfrak{a}_{\mathrm{C}}^{*}$ a basis for the solution space of (4) on $A^{+}$using the so-called Harish-Chandra series.

The Harish-Chandra series $\Phi(m ; \lambda, a)$ is defined as a formal power series on $A^{+}$with the prescribed asymptotic behavior $e^{(\lambda-\rho)(\log a)}$ :

$$
\Phi(m ; \lambda, a)=e^{(\lambda-\rho)(\log a)} \sum_{\mu \in 2 \Lambda} \Gamma_{\mu}(m ; \lambda) e^{-\mu(\log a)}, \quad a \in A^{+} .
$$

Here $\Lambda:=\left\{\sum_{j=1}^{l} n_{j} \alpha_{j}: n_{j} \in \mathrm{N}_{0}\right\}$ is the positive semigroup generated by the fundamental system of simple roots $\Pi:=\left\{\alpha_{1}, \ldots, \alpha_{l}\right\}$ in $\Sigma^{+}$. For $\mu \in 2 \Lambda \backslash\{0\}$, the coefficients $\Gamma_{\mu}(m ; \lambda)$ are rational functions of $\lambda \in a_{C}^{*}$ determined from the recursion relations

$$
\langle\mu, \mu-2 \lambda\rangle \Gamma_{\mu}(m ; \lambda)=2 \sum_{\alpha \in \Sigma^{+}} m_{\alpha} \sum_{\substack{k \in N \\ \mu-2 k \alpha \in \Lambda}} \Gamma_{\mu-2 k \alpha}(m ; \lambda)\langle\mu+\rho-2 k \alpha-\lambda, \alpha\rangle,
$$


with initial condition $\Gamma_{0}(m ; \lambda)=1$, which are derived by formally inserting the series for $\Phi$ into the differential equation (4) corresponding to $p=p_{L}$. The relations yield unique solutions $\Gamma_{\mu}(m ; \lambda)$ provided $\langle\mu, \mu-2 \lambda\rangle \neq 0$ for all $\mu \in 2 \Lambda$. For these $\lambda \in a_{\mathrm{c}}^{*}$, the series converges absolutely and uniformly on compact subsets of $A^{+}$. In fact, the Harish-Chandra series extends as a holomorphic function of $\lambda$ on $\mathfrak{a}_{\mathrm{c}}^{*} \backslash P$. Moreover, for $\lambda \in a_{\mathrm{C}}^{*} \backslash P$ one can use Harish-Chandra series to build a basis for solution space of the entire hypergeometric system with spectral parameter $\lambda$.

THEOREM 2.1. (a) ([15], Corollary 2.3) There is a connected and simply connected open subset $U$ of $T$ containing the identity element e so that the Harish-Chandra series $\Phi(m ; \lambda, h)$ extends as a meromorphic function of $(m, \lambda, h) \in \mathscr{M} \times \mathfrak{a}_{\mathrm{C}}^{*} \times A^{+} U$. Its singularities are at most simple poles located along the hyperplanes of the form $\mathscr{M} \times \mathscr{H}_{n, \alpha} \times A^{+} U$, where

$$
\mathscr{H}_{n, \alpha}:=\left\{\lambda \in \mathfrak{a}_{C}^{*}: \lambda_{\alpha}=n\right\}
$$

is a complex hyperplane in $\mathrm{a}_{\mathrm{C}}^{*}$ corresponding to some $\alpha \in \Sigma_{\mathrm{i}}^{+}$and $n \in \mathrm{N}$.

(b) ([6], Corollary 4.2.6) If $\lambda \in \mathfrak{a}_{C}^{*}$ is generic, then $\{\Phi(m ; w \lambda, a): w \in W\}$ is a basis of the solution space on $A^{+} U$ of the hypergeometric system (4) with spectral parameter $\lambda$.

Let $\Theta$ denote an arbitrary subset of the fundamental system of positive simple roots $\Pi=\left\{\alpha_{1}, \ldots, \alpha_{l}\right\}$. The set $\langle\Theta\rangle$ of elements in $\Sigma$ which can be written as linear combinations of elements from $\Theta$ is a subsystem of $\Sigma$. Its Weyl group $W_{\Theta}$ is generated by the reflections $r_{j}:=r_{\alpha_{j}}$ with $\alpha_{j} \in \Theta$. We denote by $\langle\Theta\rangle_{\mathrm{i}}$ and $\langle\Theta\rangle_{\mathrm{u}}$ respectively the indivisible and unmultipliable roots in $\langle\Theta\rangle$. Their subset of positive roots are indicated with $\langle\Theta\rangle_{i}^{+}$and $\langle\Theta\rangle_{u}^{+}$. Then
$W_{\Theta}\left(\Sigma^{+} \backslash\right.$
$\left.\langle\Theta\rangle^{+}\right) \subset \Sigma^{+} \backslash\langle\Theta$
$\langle\Theta\rangle^{+}$and
$W_{\Theta}\left(\Sigma_{\mathrm{i}}^{+} \backslash\right.$
$\left.\langle\Theta\rangle_{\mathrm{i}}^{+}\right) \subset \Sigma_{\mathrm{i}}^{+} \backslash\langle\Theta\rangle_{\mathrm{i}}^{+}$.

As in [11] and [18], we introduce the following $c$-functions. For a multiplicity function $m$ on $\Sigma$, a root $\alpha \in \Sigma^{+}$and $\lambda \in \mathfrak{a}_{\mathrm{C}}^{*}$ we set

$$
\begin{aligned}
c_{\alpha}^{+}(m ; \lambda) & :=\frac{\Gamma\left(\lambda_{\alpha}+\frac{m_{\alpha / 2}}{4}\right)}{\Gamma\left(\lambda_{\alpha}+\frac{m_{\alpha / 2}}{4}+\frac{m_{\alpha}}{2}\right)}, \\
c_{\alpha}^{-}(m ; \lambda) & :=\frac{\Gamma\left(-\lambda_{\alpha}-\frac{m_{\alpha / 2}}{4}-\frac{m_{\alpha}}{2}+1\right)}{\Gamma\left(-\lambda_{\alpha}-\frac{m_{\alpha / 2}}{4}+1\right)} .
\end{aligned}
$$


Define $c_{\Theta}^{ \pm}$and $c_{\Theta}^{ \pm, c}$ by

$$
\begin{aligned}
& \text { (6) } c_{\Theta}^{+}(m ; \lambda):=\prod_{\alpha \in\langle\Theta\rangle^{+}} c_{\alpha}^{+}(m ; \lambda), \quad c_{\Theta}^{-}(m ; \lambda):=\prod_{\alpha \in \Sigma^{+} \backslash\langle\Theta\rangle^{+}} c_{\alpha}^{-}(m ; \lambda), \\
& \text { (7) } c_{\Theta}^{-, c}(m ; \lambda):=\prod_{\alpha \in\langle\Theta\rangle^{+}} c_{\alpha}^{-}(m ; \lambda), \quad c_{\Theta}^{+, c}(m ; \lambda):=\prod_{\alpha \in \Sigma^{+} \backslash\langle\Theta\rangle^{+}} c_{\alpha}^{+}(m ; \lambda),
\end{aligned}
$$

with the conventions

$$
c_{\emptyset}^{+} \equiv c_{\Pi}^{+, c}:=1 \quad \text { and } \quad c_{\Pi}^{-} \equiv c_{\emptyset}^{-, c}:=1 .
$$

The $W$-invariance of $m$ and (5) imply that $c_{\Theta}^{-}(m ; \lambda)$ and $c_{\Theta}^{+, c}(m ; \lambda)$ are $W_{\Theta}$ invariant functions of $\lambda \in \mathfrak{a}_{\mathrm{C}}^{*}$. When $\alpha / 2$ is not a root (e.g. when $\Sigma$ is reduced), then

$$
\begin{aligned}
c_{\alpha}^{+}(m ; \lambda) & =\frac{\Gamma\left(\lambda_{\alpha}\right)}{\Gamma\left(\lambda_{\alpha}+m_{\alpha} / 2\right)} \\
c_{\alpha}^{-}(m ; \lambda) & =\frac{\Gamma\left(-\lambda_{\alpha}-m_{\alpha} / 2+1\right)}{\Gamma\left(-\lambda_{\alpha}+1\right)} .
\end{aligned}
$$

DEFINITION 2.2 ([11] and [18]). Let $\Theta \subset \Pi$ be any subset of simple roots and let $U$ be the open subset of $T$ from Theorem 2.1. The function on $A^{+} U$ defined for generic $\lambda \in a_{c}^{*}$ by

$$
\varphi_{\Theta}(m ; \lambda, h):=c_{\Theta}^{-}(m ; \lambda) \sum_{w \in W_{\Theta}} c_{\Theta}^{+}(m ; w \lambda) \Phi(m ; w \lambda, h), \quad h \in A^{+} U
$$

is called the $\Theta$-spherical function of spectral parameter $\lambda$.

As a linear combination of the Harish-Chandra series $\Phi(m ; w \lambda, h)$, the $\Theta$ spherical function of spectral parameter $\lambda$ is by construction a solution of the hypergeometric system (4) of spectral parameter $\lambda$.

EXAMPLE 2.3. When $\Theta=\Pi$, then

$$
\varphi_{\Pi}(m ; \lambda, h)=\sum_{w \in W} c_{\Pi}^{+}(m ; w \lambda) \Phi(m ; w \lambda, h)
$$

has been first considered by Heckman and Opdam in [5]. The hypergeometric function associated with the root system $\Sigma$ is

$$
F(m ; \lambda, h):=\frac{\varphi_{\Pi}(m ; \lambda, h)}{c_{\Pi}^{+}(m ; \rho(m))}=\sum_{w \in W} c(m ; w \lambda) \Phi(m ; w \lambda, h),
$$

where $c(m ; \lambda)$ denotes Harish-Chandra's $c$-function. The function $F(m ; \lambda, h)$ is normalized so that $F(m ; \lambda, e)=1$, in analogy to the classical requirement 
imposed on the spherical functions on Riemannian symmetric spaces. For geometric multiplicities $m$, the hypergeometric functions $F(m ; \lambda, h)$ agree with Harish-Chandra's spherical functions. In particular, $\varphi_{\text {П }}(m ; \lambda, h)$ reduces to a spherical function up to a multiplicative factor depending only on the multiplicities.

ExAmple 2.4. Suppose $\Sigma$ is the restricted root system of a NCC symmetric space (in particular $\Sigma$ is reduced). Let $\Pi_{0}$ be the fundamental system for the positive compact roots $\Sigma_{0}^{+}$and set $\Theta:=\Pi_{0}$. Then $\langle\Theta\rangle=\Sigma_{0}$, and $W_{\Theta}=W_{0}$ is the so-called small Weyl group. Up to a factor depending only on the multiplicities, the $\Theta$-spherical functions reduce to Unterberger's [19] for arbitrary multiplicities, and to the spherical functions on NCC symmetric spaces for geometric multiplicities.

EXAmPle 2.5. When $\Theta=\emptyset$, then $W_{\emptyset}=\{\mathrm{id}\}$, hence $\varphi_{\emptyset}(m ; \lambda, h)=$ $c_{\emptyset}^{-}(m ; \lambda) \Phi(m ; \lambda, h)$.

The function $\varphi_{\Theta}$ is a priori defined only for $\lambda \in a_{\mathrm{C}}^{*}$ generic and $h \in A^{+} U$. In the case $\Theta=\Pi$, Heckman and Opdam proved that $\varphi_{\Pi}(m ; \lambda, h)$ extends as a $W$-invariant holomorphic function in $\mathcal{M} \times \mathrm{a}_{\mathrm{C}}^{*} \times U_{\Pi}$, where $U_{\text {п }}$ denotes a $W$ invariant tubular neighborhood of $A$ in $A_{C}$ (see e.g. [6], §4.3-4.4). Their result parallels the regularity properties of the spherical functions on Riemannian symmetric spaces. For arbitrary $\Theta \subset \Pi$, there exists a certain $W_{\Theta}$-invariant open domain $U_{\Theta} \subset A_{\subset}$ such that the right-hand side of (8) extends as a $W_{\Theta}$ invariant holomorphic function of $h \in U_{\Theta}$, as a $W_{\Theta}$-invariant meromorphic function of $\lambda \in \mathfrak{a}_{\mathrm{C}}^{*}$ and as entire function of $m \in \mathcal{M}$. See Theorem 2.6 below. The domain $U_{\Theta}$ is a tubular neighborhood in $A_{\subset}$ of an open domain $A_{\Theta}$ constructed as follows. Let $\overline{a^{+}}$denote the closure of the positive Weyl chamber, and let $\overline{A^{+}}:=\exp \left(\overline{\mathfrak{a}^{+}}\right)$. We define

$$
\mathfrak{a}_{\Theta}:=W_{\Theta}\left(\overline{\mathfrak{a}^{+}}\right)^{0} \quad \text { and } \quad A_{\Theta}:=\exp \mathfrak{a}_{\Theta}=W_{\Theta}\left(\overline{A^{+}}\right)^{0},
$$

where ${ }^{0}$ denotes the interior. Then $\mathfrak{a}_{\Theta}$ is a $W_{\Theta}$-invariant open convex cone (see [18], Lemma 1.5.10).

The duplication formula for the gamma function

$$
\sqrt{\pi} \Gamma(2 z)=2^{2 z-1} \Gamma(z) \Gamma(z+1 / 2)
$$

yields

$$
c_{\Theta}^{-}(m ; \lambda)=\prod_{\alpha \in \Sigma_{i}^{+} \backslash\langle\Theta\rangle_{i}^{+}} \frac{1}{\sqrt{\pi}} \frac{\Gamma\left(-\frac{\lambda_{\alpha}}{2}-\frac{m_{\alpha}}{4}+\frac{1}{2}\right) \Gamma\left(-\frac{\lambda_{\alpha}}{2}-\frac{m_{\alpha}}{4}-\frac{m_{2 \alpha}}{2}+1\right)}{2^{\lambda_{\alpha}+m_{\alpha} / 2} \Gamma\left(-\lambda_{\alpha}+1\right)},
$$


which justifies the definition of

$$
n_{\Theta}^{-}(m ; \lambda):=\prod_{\alpha \in \Sigma_{i}^{+} \backslash\langle\Theta\rangle_{i}^{+}} \Gamma\left(-\frac{\lambda_{\alpha}}{2}-\frac{m_{\alpha}}{4}+\frac{1}{2}\right) \Gamma\left(-\frac{\lambda_{\alpha}}{2}-\frac{m_{\alpha}}{4}-\frac{m_{2 \alpha}}{2}+1\right)
$$

$$
d_{\Theta}^{-}(m ; \lambda):=\prod_{\alpha \in \Sigma_{i}^{+} \backslash\langle\Theta\rangle_{i}^{+}} 2^{\lambda_{\alpha}+m_{\alpha} / 2} \sqrt{\pi} \Gamma\left(-\lambda_{\alpha}+1\right)
$$

as the numerator, respectively the denominator, of $c_{\Theta}^{-}(m ; \lambda)$. When $2 \alpha \notin \Sigma^{+}$ for all $\alpha \in \Sigma_{\mathrm{i}}^{+} \backslash\langle\Theta\rangle_{\mathrm{i}}^{+}$(e.g. when $\Sigma$ is reduced), the duplication formula (9) implies

$$
n_{\Theta}^{-}(m ; \lambda)=\prod_{\alpha \in \Sigma^{+} \backslash\langle\Theta\rangle^{+}} 2^{\lambda_{\alpha}+m_{\alpha} / 2} \sqrt{\pi} \Gamma\left(-\lambda_{\alpha}-\frac{m_{\alpha}}{2}+1\right) .
$$

The regularity properties of the $\Theta$-spherical functions are collected in the following theorem.

Theorem 2.6. ([11], Theorem 8.3, or [18], Theorem 3.5) There exists a $W_{\Theta}$-invariant tubular neighborhood $U_{\Theta}$ of $A_{\Theta}$ in $A_{C}$ such that the function

$$
\frac{\varphi_{\Theta}(m ; \lambda, h)}{n_{\Theta}^{-}(m ; \lambda)}=\frac{1}{d_{\Theta}^{-}(m ; \lambda)} \sum_{w \in W_{\Theta}} c_{\Theta}^{+}(m ; w \lambda) \Phi(m ; w \lambda ; h)
$$

extends as a holomorphic function of $(m, \lambda, h) \in \mathcal{M} \times \mathfrak{a}_{\mathrm{C}}^{*} \times U_{\Theta}$ and satisfies

$$
\frac{\varphi_{\Theta}(m ; w \lambda, h)}{n_{\Theta}^{-}(m ; w \lambda)}=\frac{\varphi_{\Theta}(m ; \lambda, h)}{n_{\Theta}^{-}(m ; \lambda)}=\frac{\varphi_{\Theta}(m ; \lambda, w h)}{n_{\Theta}^{-}(m ; \lambda)}
$$

for all $w \in W_{\Theta}$ and $(m, \lambda, h) \in \mathscr{M} \times \mathfrak{a}_{\subset}^{*} \times U_{\Theta}$. In particular, the $\lambda$-singularities of the $\Theta$-spherical function $\varphi_{\Theta}(m ; \lambda, h)$ are contained (counted with multiplicities) in the polar set of the numerator function $n_{\Theta}^{-}(m ; \lambda)$. The $\lambda$-singularities are at most simple poles when $\Sigma$ is a reduced root system.

Furthermore, the function

$$
\frac{\varphi_{\Theta}(m ; \lambda, h)}{c_{\Theta}^{-}(m ; \lambda)}
$$

is holomorphic in $\lambda$ on $B_{\Theta}:=\left\{\lambda \in \mathfrak{a}_{\mathrm{C}}^{*}: \operatorname{Re} \lambda_{\alpha}<1\right.$ for all $\left.\alpha \in \Sigma_{\mathrm{i}}^{+} \backslash\langle\Theta\rangle_{\mathrm{i}}^{+}\right\}$.

When $\Sigma$ is reduced and $m \in \mathscr{M}^{+}$is an even multiplicity function, then the $\lambda$ singularities of the $\Theta$-spherical functions are indeed located only along finitely 
many complex hyperplanes. We refer to [12], Section 3, for more information on this special case.

For $\Theta \neq \Pi$, the $\Theta$-spherical functions are singular in the space variable $a$ along the "walls" of $A_{\Theta}$. An upper bound for the order of singularity is provided by the next theorem in the case $m \in \mathscr{M}^{+}$.

For $R>0$, let

$$
\mathfrak{a}_{\mathrm{C}}^{*}(R):=\left\{\lambda \in \mathfrak{a}_{\mathrm{C}}^{*}: \operatorname{Re}\langle\lambda, \alpha\rangle<R \text { for all } \alpha \in \Sigma^{+}\right\} .
$$

Since only finitely many singular hyperplanes intersect $a_{\mathrm{C}}^{*}(R)$, the $\lambda$-singularities of $\varphi_{\Theta}$ in $\mathfrak{a}_{C}^{*}(R)$ are canceled by multiplication by a polynomial function which is a finite product of linear factors in $\langle\lambda, \alpha\rangle$ with $\alpha \in \Sigma^{+}$. We set

$$
\delta(m ; a):=\prod_{\alpha \in \Sigma^{+}}\left|e^{\alpha(\log a)}-e^{-\alpha(\log a)}\right|^{m_{\alpha}}
$$

for all $a \in A$.

Theorem 2.7. Suppose $m \in \mathscr{M}^{+}$. Assume that $m_{\alpha}>0$ for all $\alpha \in \Sigma$, and set $m_{2 \alpha}=0$ if $2 \alpha \notin \Sigma$. Let $R>0$ and let $\mathrm{a}_{\mathrm{C}}^{*}(R)$ be as in (12).

(a) ([18], Theorem 4.17 and Lemma 4.11) There exists $c \in[0,1)$ (depending only on the multiplicity function $m$ and explicitly computable) such that for a polynomial $p_{R}$ and and a constant $C_{R, m}>0$

$\left|p_{R}(\lambda) \Phi(m ; \lambda, a)\right| \delta(m ; a)^{(c+1) / 2} \leq C_{R, m}(1+|\lambda|)^{\operatorname{deg} p_{R}} e^{(c \rho(m)+\operatorname{Re} \lambda)(\log a)}$

for all $a \in A^{+}$and $\lambda \in \mathrm{a}_{\mathrm{C}}^{*}(R)$. Moreover there is $R_{0}>0$ such that we can choose $p_{R} \equiv 1$ for all $R$ with $0<R<R_{0}$.

(b) ([16], Theorem 3.15) For all $a \in A$ and $\lambda \in a_{C}^{*}$

$$
\left|\varphi_{\Pi}(m ; \lambda, a)\right| \leq|W|^{1 / 2} c_{\Pi}^{+}(m ; \rho(m)) e^{\max _{w \in W} \operatorname{Re}(w \lambda(\log a))} .
$$

(c) ([18], Theorem 5.5) Let c be as in (a). For $a \in A_{\Theta}$, let $a_{\Theta} \in W_{\Theta} a \cap \overline{A^{+}}$ be the unique element of the $W_{\Theta}$-orbit of a lying in $\overline{A^{+}}$. Moreover, set

$$
\operatorname{Re} W_{\Theta} \lambda(\log a):=\max _{w \in W_{\Theta}} \operatorname{Re} w \lambda(\log a)
$$

for all $\lambda \in \mathfrak{a}_{C}^{*}$. Then there exists a polynomial $p_{\Theta, R, m}(\lambda)$ and constants $C_{\Theta, R, m}>0, d(\Theta, R, m) \geq 0$ such that

$$
\begin{aligned}
\left|p_{\Theta, R, m}(\lambda) \varphi_{\Theta}(m ; \lambda, a)\right| & \delta(m ; a)^{(c+1) / 2} \\
& \leq C_{\Theta, R, m}(1+|\lambda|)^{d(\Theta, R, m)} e^{\left(c \rho(m)+\operatorname{Re} W_{\Theta} \lambda\right)\left(\log a_{\Theta}\right)}
\end{aligned}
$$


for all $a \in A_{\Theta}$ and $\lambda \in \mathfrak{a}_{\mathrm{C}, \Theta}^{*}(R)$.

\section{The $\Theta$-spherical transform}

In this section we define the $\Theta$-spherical transform of $W_{\Theta}$-invariant functions. Here and in the following we suppose that $m \in \mathscr{M}^{+}$. Under this assumption, the function $\delta(m ; a)$ in (13) is continuous and $W$-invariant in $a \in A$.

Definition 3.1. Let $\Theta \subset \Pi$ and let $f$ be a $W_{\Theta}$-invariant function on $A_{\Theta}=W_{\Theta}\left(\overline{A^{+}}\right)^{0}$. The $\Theta$-spherical Fourier transform of $f$ associated with the data $(\mathfrak{a}, \Sigma, m)$ is the $W_{\Theta}$-invariant function $\mathscr{F}_{\Theta} f(m)$ on $\mathfrak{a}_{\mathcal{C}}^{*}$ defined for $\lambda \in \mathfrak{a}_{\mathcal{C}}^{*}$ by

$$
\begin{aligned}
\mathscr{F}_{\Theta} f(m ; \lambda) & :=\frac{1}{\left|W_{\Theta}\right|} \int_{A_{\Theta}} f(a) \varphi_{\Theta}(m ; \lambda, a) \delta(m ; a) d a \\
& =\int_{A^{+}} f(a) \varphi_{\Theta}(m ; \lambda, a) \delta(m ; a) d a,
\end{aligned}
$$

provided the integral converges. Here $d a$ is a suitable normalization of the Haar measure on $A$.

EXAMPLE 3.2. When $\Theta=\Pi$, then $\mathscr{F}_{\Theta}$ agrees with the transform of [16] (see also [6], p. 205). In particular, for geometric multiplicities, we recover HarishChandra's spherical transform for $K$-bi-invariant functions on the Riemannian space $G / K$.

Example 3.3. Suppose $\Sigma$ is the restricted root system of a NCC symmetric space, and let $\Theta=\Pi_{0}$ be as in Example 2.4. For geometric multiplicities the $\Theta$-spherical transform is the spherical Laplace transform of [1] and [10]. To our knowledge, the $\Theta$-spherical transform for $\Theta=\Pi_{0}$ and arbitrary nongeometric multiplicities does not appear in the literature.

For $\Theta \neq \Pi$, the $\Theta$-spherical functions $\varphi_{\Theta}(m ; \lambda, a)$ are singular along the "walls" of the domain $A_{\Theta}$, but the estimates of Theorem 2.7(c) ensure that (for almost all $\left.\lambda \in \mathfrak{a}_{\mathrm{C}}^{*}\right)$ the function $\varphi_{\Theta}(m ; \lambda, a) \delta(m ; a)$ vanishes along the walls of $A_{\Theta}$. Hence, as in the case $\Theta=\Pi$, the defining integral for $\mathscr{F}_{\Theta} f(m ; \lambda)$ converges under the only condition of sufficiently fast decay of $f$ at infinity on $A_{\ominus}$. In fact, since $c \in[0,1)$, we can even admit some singularity for $f$ along the walls of $A_{\Theta}$. Applied to functions which are compactly supported in $\overline{A_{\Theta}}$, the $\Theta$-spherical transform inherits the regularity properties in $\lambda \in \mathfrak{a}_{\subset}^{*}$ of the $\Theta$-spherical functions. Indeed, let $C_{c}\left(\overline{A_{\Theta}}\right)^{W_{\Theta}}$ denote the set of $W_{\Theta}$-invariant continuous functions on $\overline{A_{\Theta}}$ with compact support. Then a classical application of the theorems of Fubini and Morera prove the following proposition. 
Proposition 3.4. The $\Theta$-spherical Fourier transform $\mathscr{F}_{\Theta} f(m ; \lambda)$ of $f \in$ $C_{c}\left(\overline{A_{\Theta}}\right)^{W_{\Theta}}$ is a $W_{\Theta}$-invariant meromorphic function of $\lambda \in \mathfrak{a}_{\mathrm{C}}^{*}$. Its singularities are located (counting multiplicities) in the polar set of the numerator function $n_{\Theta}^{-}(m ; \lambda)$ defined by formula $(10)$. When $\Sigma$ is reduced, then all singularities are simple poles.

In [10], Ólafsson proved that the spherical functions on a NCC symmetric space $G / H$, initially defined by means of an integral formula for all $\lambda$ in a certain domain in $a_{C}^{*}$, admit on $A^{+}$an expansion of the form (8). Using the classical expansion of the the spherical functions on the Riemannian dual symmetric space $G / K$ in terms of the Harish-Chandra series, he deduced a functional relation expressing the spherical functions on $G / K$ in terms of the spherical functions on $G / H$. Our definition of $\Theta$-spherical functions allows us to easily extend his functional relation to our context. In fact, Ólafsson's expansion and functional relation hold even in some tubular neighborhood of $A_{\Theta}$ in $A_{\mathrm{C}}$.

LeMma 3.5. There is a $W_{\Theta}$-invariant tubular neighborhood $U_{\Theta}$ of $A_{\Theta}$ in $A_{C}$ so that for all $(m, \lambda, h) \in \mathscr{M} \times \mathfrak{a}_{\mathrm{C}}^{*} \times U_{\Theta}$ the following equality of meromorphic functions holds:

$$
\varphi_{\Pi}(m ; \lambda, h)=\sum_{w \in W_{\Theta} \backslash W} \frac{c_{\Theta}^{+, c}(m ; w \lambda)}{c_{\Theta}^{-}(m ; w \lambda)} \varphi_{\Theta}(m ; w \lambda, h),
$$

where the functions $c_{\Theta}^{+, c}(m ; \lambda)$ and $c_{\Theta}^{-}(m ; \lambda)$ are as in (6) and (7).

Proof. By $W_{\Theta}$-invariance in $\lambda$ of $c_{\Theta}^{+, c}(m ; \lambda), c_{\Theta}^{-}(m ; \lambda)$ and $\varphi_{\Theta}(m ; \lambda, a)$, we obtain for all $m \in \mathscr{M}, h \in A^{+} U$ and for generic $\lambda \in \mathfrak{a}_{\mathrm{C}}^{*}$

$$
\begin{aligned}
\varphi_{\Pi}(m ; \lambda, h) \\
:=\sum_{w \in W} c_{\Pi}^{+}(m ; w \lambda) \Phi(m ; w \lambda, h) \\
=\sum_{w \in W_{\Theta} \backslash W} \sum_{w^{\prime} \in W_{\Theta}} c_{\Theta}^{+}\left(m ; w^{\prime} w \lambda\right) c_{\Theta}^{+, c}\left(m ; w^{\prime} w \lambda\right) \Phi\left(m ; w^{\prime} w \lambda, h\right) \\
=\sum_{w \in W_{\Theta} \backslash W} \frac{c_{\Theta}^{+, c}(m ; w \lambda)}{c_{\Theta}^{-}(m ; w \lambda)}\left(c_{\Theta}^{-}(m ; w \lambda) \sum_{w^{\prime} \in W_{\Theta}} c_{\Theta}^{+}\left(m ; w^{\prime} w \lambda\right) \Phi\left(m ; w^{\prime} w \lambda, h\right)\right) \\
=\sum_{w \in W_{\Theta} \backslash W} \frac{c_{\Theta}^{+, c}(m ; w \lambda)}{c_{\Theta}^{-}(m ; w \lambda)} \varphi_{\Theta}(m ; w \lambda, h) .
\end{aligned}
$$

The equality extends by analyticity to $(m, \lambda, h) \in \mathscr{M} \times \mathfrak{a}_{\mathrm{C}}^{*} \times U_{\Theta}$ for a suitable $W_{\Theta}$-invariant tubular neighborhood $U_{\Theta}$ of $A_{\Theta}$ in $A_{\mathrm{C}}$. 
Let $C_{c}\left(A_{\Theta}\right)^{W_{\Theta}}$ denote the space of continuous $W_{\Theta}$-invariant functions on $A_{\Theta}$ with compact support, and let $C_{c}^{\infty}\left(A_{\Theta}\right)^{W_{\Theta}}$ denote the subspace of $C^{\infty}$ functions. Every $f \in C_{c}\left(A_{\Theta}\right)^{W_{\Theta}}$ (resp., $f \in C_{c}^{\infty}\left(A_{\Theta}\right)^{W_{\Theta}}$ ) extends uniquely to a $W$-invariant compactly supported continuous (resp., $C^{\infty}$ ) function on $A$, which we still denote by $f$. The relations among $\Theta$-spherical functions of Lemma 3.5 yield the following relations for the associated $\Theta$-spherical transforms.

Corollary 3.6. For every $f \in C_{c}\left(A_{\Theta}\right)^{W_{\Theta}}$ we have the following equality of meromorphic functions of $\lambda \in \mathfrak{a}_{\mathrm{C}}^{*}$ :

$$
\mathscr{F}_{\Pi} f(m ; \lambda)=\sum_{w \in W_{\Theta} \backslash W} \frac{c_{\Theta}^{+, c}(m ; w \lambda)}{c_{\Theta}^{-}(m ; w \lambda)} \mathscr{F}_{\Theta} f(m ; w \lambda) .
$$

\section{Inversion of the $\Theta$-spherical Fourier transform}

In this section we establish the inversion formula for the $\Theta$-spherical Fourier transform on the space $C_{c}^{\infty}\left(A_{\Theta}\right)^{W_{\Theta}}$ of $W_{\Theta}$-invariant compactly supported $C^{\infty}$ functions on $A_{\Theta}$. The method employed generalizes the procedure followed by Ólafsson [10] in the case of the spherical transform on noncompactly causal symmetric spaces, which is a reduction to the inversion of the Harish-Chandra's spherical transform on the dual Riemannian symmetric space. Similarly, we shall deduce the inversion of the $\Theta$-spherical Fourier transform from the inversion of the П-spherical Fourier transform, which is due to Opdam [16]. The reduction is obtained via Corollary 3.6. As in the previous sections, we always consider multiplicity functions $m \in \mathcal{M}^{+}$with $m_{\alpha}>0$ for all $\alpha \in \Sigma$, and set $m_{2 \alpha}=0$ if $2 \alpha \notin \Sigma$.

Let $\Theta \subset \Pi$ be fixed. The following lemma rephrases in our notation some very well-known properties of the Harish-Chandra's $c$-function for imaginary values of the spectral parameter $\lambda$ (see e.g. [2], Propositions 4.7.14 and 4.7.15).

LEMMA 4.1. The functions $c_{\Theta}^{ \pm}$from (6) and (7) satisfy the following properties:

(a) $c_{\Theta}^{ \pm}(m ;-i \lambda)=c_{\Theta}^{ \pm}(m ; \overline{i \lambda})=\overline{c_{\Theta}^{ \pm}(m ; i \lambda)}$ for all $\lambda \in a^{*}$, where - denotes complex conjugation.

(b) $c_{\Theta}^{ \pm}(m ; \lambda) c_{\Theta}^{ \pm}(m ;-\lambda)$ is $W_{\Theta}$-invariant.

(c) $\left|c_{\Theta}^{ \pm}(m ; i \lambda)\right|$ is $W_{\Theta}$-invariant for all $\lambda \in \mathfrak{a}^{*}$.

(d) $1 / c_{\Theta}^{+}(m ; \lambda)$ is a holomorphic function of $\lambda$ in the open neighborhood

$$
\begin{aligned}
B_{\Theta, m}:=\left\{\lambda \in a_{\mathrm{C}}^{*}\right. & : \operatorname{Re} \lambda_{\alpha} \\
& \left.>\max \left\{-m_{\alpha} / 2-1,-m_{\alpha} / 2-m_{2 \alpha}\right\} \text { for all } \alpha \in\langle\Theta\rangle_{i}^{+}\right\}
\end{aligned}
$$


of $i \mathfrak{a}^{*}$ in $\mathfrak{a}_{\mathrm{c}}^{*}$.

(e) There are constants $C_{m}>0$ and $r(m) \geq 0$ such that

$$
\left|c_{\Theta}^{+}(m ;-i \lambda)\right|^{-1} \leq C_{m}(1+|\lambda|)^{r(m)}
$$

for all $\lambda \in \mathfrak{a}^{*}$. $\overline{A^{+}}$.

Recall the notation $a_{\Theta}$ for the element of the $W_{\Theta}$-orbit of $a \in A_{\Theta}$ lying in

Lemma 4.2. Let $m \in \mathscr{M}^{+}$, and let $c \in[0,1)$ be the constant occurring in the estimates of Theorem 2.7. Let $B_{\Theta}$ and $B_{\ominus, m}$ be the open neighborhoods of $i a^{*}$ in $a_{C}^{*}$ respectively introduced in Theorem 2.7 and in part (d) of Lemma 4.1. Define

$$
c_{\Theta}(m ; \lambda):=c_{\Theta}^{+}(m ; \lambda) c_{\Theta}^{-}(m ; \lambda) .
$$

Then for every $a \in A_{\oplus}$ the function

$$
\frac{\varphi_{\Theta}(m ; \lambda, a)}{c_{\Theta}(m ; \lambda)}
$$

is holomorphic in $\lambda \in B_{\Theta} \cap B_{\Theta, m}$. Moreover, there is a constant $C_{m}>0$ such that

$$
\delta(m ; a)\left|\frac{\varphi_{\Theta}(m ; i \lambda, a)}{c_{\Theta}(m ; i \lambda)}\right| \leq C_{m}\left|W_{\Theta}\right| \delta(m ; a)^{(1-c) / 2} e^{c \rho(m)\left(\log a_{\Theta}\right)}
$$

for all $a \in A_{\Theta}$ and $\lambda \in \mathfrak{a}^{*}$.

Proof. The holomorphy of $\varphi_{\Theta}(m ; \lambda, a) / c_{\Theta}(m ; \lambda)$ in $B_{\Theta} \cap B_{\Theta, m}$ follows immediately from the last statement in Theorem 2.6 and Lemma 4.1(d).

According to (8), we have for all $a \in A^{+}$and generic $\lambda \in \mathfrak{a}^{*}$

$$
\frac{\varphi_{\Theta}(m ; i \lambda, a)}{c_{\Theta}(m ; i \lambda)}=\sum_{w \in W_{\Theta}} \frac{c_{\Theta}^{+}(m ; i w \lambda)}{c_{\Theta}^{+}(m ; i \lambda)} \Phi(m ; i w \lambda, a) .
$$

Notice that $i \mathfrak{a}^{*} \subset \mathfrak{a}_{\mathrm{C}}^{*}(R)$ for all $R>0$. We can therefore fix $R>0$ small enough in Theorem 2.7 so that the polynomial $p_{R}$ is identically equal to 1 . Observing moreover that

$$
\left|\frac{c_{\Theta}^{+}(m ; i w \lambda)}{c_{\Theta}^{+}(m ; i \lambda)}\right|=1
$$


by part (c) of Lemma 4.1, we obtain

$$
\begin{aligned}
\delta(m ; a)\left|\frac{\varphi_{\Theta}(m ; i \lambda, a)}{c_{\Theta}(m ; i \lambda)}\right| & \leq \sum_{w \in W_{\Theta}} \delta(m ; a)|\Phi(m ; i w \lambda, a)| \\
& \leq C_{m, c} \sum_{w \in W_{\Theta}} \delta(m ; a)^{(1-c) / 2} e^{(c \rho(m)+\operatorname{Re} i w \lambda)(\log a)} \\
& =C_{m, c}\left|W_{\Theta}\right| \delta(m ; a)^{(1-c) / 2} e^{c \rho(m)\left(\log a_{\Theta}\right)}
\end{aligned}
$$

The inequality extends by $W_{\Theta}$-invariance to $A_{\Theta}$ and by continuity to all $\lambda \in \mathfrak{a}^{*}$.

Corollary 4.3. For every $f \in C_{c}^{\infty}\left(A_{\ominus}\right)^{W_{\ominus}}$, the function

$$
\frac{\mathscr{F}_{\Theta} f(m ; i \lambda)}{c_{\Theta}(m ; i \lambda)}
$$

is real analytic in $\lambda \in \mathfrak{a}^{*}$. Moreover, for every $N \in \mathbf{N}$ there is a constant $C_{\Theta, N, m, f}>0$ such that

$$
\left|\frac{\mathscr{F}_{\Theta} f(m ; i \lambda)}{c_{\Theta}(m ; i \lambda)}\right| \leq C_{\Theta, N, m, f}(1+|\lambda|)^{-N}
$$

for all $\lambda \in \mathfrak{a}^{*}$.

Proof. For every $a \in A_{\Theta}$, the function $\varphi_{\Theta}(m ; \lambda, a) / c_{\Theta}(m ; \lambda)$ is holomorphic in $B_{\Theta} \cap B_{\Theta, m}$ and $f$ is continuous and compactly supported in $A_{\ominus}$. The usual application of the theorems of Fubini and Morera yields that $\mathscr{F}_{\Theta} f(m ; \lambda) /$ $c_{\Theta}(m ; \lambda)$ is holomorphic in $\lambda \in B_{\Theta} \cap B_{\Theta, m}$. In particular, its restriction to $i \mathfrak{a}^{*}$ is real analytic.

By [6], Theorem 2.1.1, the operator $L(m)$ is self-adjoint with respect to the measure $\delta(m ; a) d a$ on $A$. Moreover for all $\lambda \in \mathfrak{a}^{*}$

$$
L(m) \varphi_{\Theta}(m ; i \lambda)=-(\langle\lambda, \lambda\rangle+\langle\rho, \rho\rangle) \varphi_{\Theta}(m ; i \lambda)=-\left(|\lambda|^{2}+|\rho|^{2}\right) \varphi_{\Theta}(m ; i \lambda),
$$

which implies

$$
L(m)^{N} \varphi_{\Theta}(m ; i \lambda)=(-1)^{N}\left(|\lambda|^{2}+|\rho|^{2}\right)^{N} \varphi_{\Theta}(m ; i \lambda)
$$

for all $N \in \mathrm{N}$. The function $f$ is $C^{\infty}$ and compactly supported in $A_{\Theta}$, so no 
boundary values occur in the following computation:

$$
\begin{aligned}
\left(|\lambda|^{2}\right. & \left.+|\rho|^{2}\right)^{N} \frac{\mathscr{F}_{\Theta} f(m ; i \lambda)}{c_{\Theta}(m ; i \lambda)} \\
& =\int_{A_{\Theta}} f(a)\left(|\lambda|^{2}+|\rho|^{2}\right)^{N} \frac{\varphi_{\Theta}(m ; i \lambda, a)}{c_{\Theta}(m ; i \lambda)} \delta(m ; a) d a \\
& =(-1)^{N} \int_{A_{\Theta}} f(a) \frac{L(m)^{N} \varphi_{\Theta}(m ; i \lambda, a)}{c_{\Theta}(m ; i \lambda)} \delta(m ; a) d a \\
& =(-1)^{N} \int_{A_{\Theta}}\left[L(m)^{N} f(a)\right] \frac{\varphi_{\Theta}(m ; i \lambda, a)}{c_{\Theta}(m ; i \lambda)} \delta(m ; a) d a .
\end{aligned}
$$

Lemma 4.2 gives for some constant $C_{\Theta, N, m, f}^{\prime}>0$

$$
\begin{aligned}
\left(|\lambda|^{2}+|\rho|^{2}\right)^{N}\left|\frac{\mathscr{F}_{\Theta} f(m ; i \lambda)}{c_{\Theta}(m ; i \lambda)}\right| & \leq \int_{A_{\Theta}}\left|L(m)^{N} f(a)\right|\left|\frac{\varphi_{\Theta}(m ; i \lambda, a)}{c_{\Theta}(m ; i \lambda)}\right| \delta(m ; a) d a \\
& \leq C_{\Theta, N, m, f}^{\prime},
\end{aligned}
$$

from which the claim follows.

For every $\lambda \in \mathfrak{a}_{\mathcal{C}}^{*}$ we define the function $E_{\Theta}(m ; \lambda)$ on $A_{\Theta}$ by requiring that the equality

$$
E_{\Theta}(m ; \lambda, a)=\frac{c_{\Theta}^{-}(m ; \lambda)}{c_{\Theta}^{+, c}(m ; \lambda)} \varphi_{\Pi}(m ; \lambda, a)
$$

holds for all $a \in A^{+}$. Then $E_{\Theta}(m ; \lambda, a)$ is a $W_{\Theta}$-invariant real analytic function of $a \in A_{\Theta}$ and a $W_{\Theta}$-invariant meromorphic function of $\lambda \in \mathfrak{a}_{\mathrm{C}}^{*}$.

Remark 4.4. Notice that $E_{\Pi}(m ; \lambda, a)=\varphi_{\Pi}(m ; \lambda, a)$. When $m$ is an even multiplicity function, we have

$$
\begin{aligned}
\frac{c_{\Theta}^{-}(m ; \lambda)}{c_{\Theta}^{+, c}(m ; \lambda)}=\prod_{\alpha \in \Sigma^{+} \backslash\langle\Theta\rangle^{+}} \frac{c_{\alpha}^{-}(m ; \lambda)}{c_{\alpha}^{+}(m ; \lambda)} & =\prod_{\alpha \in \Sigma^{+} \backslash\langle\Theta\rangle^{+}} \frac{\sin \left(\pi \lambda_{\alpha}\right)}{\sin \left[\pi\left(\lambda_{\alpha}+m_{\alpha} / 2\right)\right]} \\
& =(-1)^{d(m, \Theta)}
\end{aligned}
$$

where $d(m, \Theta):=\frac{1}{2} \sum_{\alpha \in \Sigma^{+} \backslash\langle\Theta\rangle^{+}} m_{\alpha}$. Hence $E_{\Theta}(m ; \lambda, a)= \pm \varphi_{\text {П }}(m ; \lambda, a)$ for all $\Theta$.

THEOREM 4.5. There is a constant $k>0$ (depending only on the normalization of the measures) so that for all $f \in C_{c}^{\infty}\left(A_{\Theta}\right)^{W_{\ominus}}$ the following inversion 
formula holds: For all $a \in A_{\Theta}$

$$
f(a)=k \frac{|W|}{\left|W_{\Theta}\right|} \int_{i a^{*}} \mathscr{F}_{\Theta} f(m ; \lambda) E_{\Theta}(m ;-\lambda, a) \frac{d \lambda}{\left|c_{\Theta}(m ; \lambda)\right|^{2}},
$$

where $d \lambda$ is a suitable normalization of the Lebesgue measure on $i \mathrm{a}^{*}$.

Proof. Let $f \in C_{c}^{\infty}\left(A_{\Theta}\right)^{W_{\Theta}}$ and extend it by $W$-invariance to a function in $C_{c}^{\infty}(A)$, which we still denote by the symbol $f$. Opdam's inversion formula for $\mathscr{F}_{\Pi}(m)$ gives for all $a \in A$ and for a constant $k>0$ depending only on the normalization of the measures

$$
f(a)=k \int_{i a^{*}} \mathscr{F}_{\Pi} f(m ; \lambda) \varphi_{\Pi}(m ;-\lambda, a) \frac{d \lambda}{\left|c_{\Pi}^{+}(m ; \lambda)\right|^{2}}
$$

(see Theorem 9.13(3) in [16] or formula (8.7) in [17]). Corollary 3.6, the $W$ invariance of $\varphi_{\Pi}(m ; \lambda, a)$ and $c_{\Pi}^{+}(m ; \lambda)$, and the properties of the functions $c_{\Theta}^{ \pm}$from Lemma 4.1 imply therefore the following formal computation for $a \in A^{+}$:

$$
\begin{aligned}
f(a) & k \int_{i \mathfrak{a}^{*}} \mathscr{F}_{\Pi} f(m ; \lambda) \varphi_{\Pi}(m ;-\lambda, a) \frac{d \lambda}{\left|c_{\Pi}^{+}(m ; \lambda)\right|^{2}} \\
= & k \sum_{w \in W_{\Theta} \backslash W} \int_{i \alpha^{*}} \mathscr{F}_{\Theta} f(m ; w \lambda) \varphi_{\Pi}(m ;-\lambda, a) \frac{c_{\Theta}^{+, c}(m ; w \lambda)}{c_{\Theta}^{-}(m ; w \lambda)} \frac{d \lambda}{c_{\Pi}^{+}(m ; \lambda) c_{\Pi}^{+}(m ;-\lambda)} \\
= & k \sum_{w \in W_{\Theta} \backslash W} \int_{i \alpha^{*}} \mathscr{F}_{\Theta} f(m ; w \lambda) \frac{\varphi_{\Pi}(m ;-w \lambda, a)}{c_{\Theta}^{-}(m ; w \lambda) c_{\Theta}^{+, c}(m ;-w \lambda)} \frac{d \lambda}{c_{\Theta}^{+}(m ; w \lambda) c_{\Theta}^{+}(m ;-w \lambda)} \\
= & k \sum_{w \in W_{\Theta} \backslash W} \int_{i \alpha^{*}} \mathscr{F}_{\Theta} f(m ; \lambda) \frac{\varphi_{\Pi}(m ;-\lambda, a)}{c_{\Theta}^{-}(m ; \lambda) c_{\Theta}^{+, c}(m ;-\lambda)} \frac{d \lambda}{c_{\Theta}^{+}(m ; \lambda) c_{\Theta}^{+}(m ;-\lambda)} \\
= & k \frac{|W|}{\left|W_{\Theta}\right|} \int_{i a^{*}} \mathscr{F}_{\Theta} f(m ; \lambda) E_{\Theta}(m ;-\lambda, a) \frac{d \lambda}{\left|c_{\Theta}(m ; \lambda)\right|^{2}} .
\end{aligned}
$$

The computation is justified provided at the first step we can interchange the integral sign with the summation. This is equivalent to verifying the convergence of the integral

$$
\int_{i a^{*}} \mathscr{F}_{\Theta} f(m ; \lambda) E_{\Theta}(m ;-\lambda, a) \frac{d \lambda}{\left|c_{\Theta}(m ; \lambda)\right|^{2}} .
$$


The absolute value of the integrand is

$$
\left|\frac{\mathscr{F}_{\Theta} f(m ; \lambda)}{c_{\Theta}(m ; \lambda)}\right|\left|\frac{c_{\Theta}^{-}(m ;-\lambda) \varphi_{\Pi}(m ;-\lambda, a)}{c_{\Theta}^{+, c}(m ;-\lambda) c_{\Theta}(m ;-\lambda)}\right|=\left|\frac{\mathscr{F}_{\Theta} f(m ; \lambda)}{c_{\Theta}(m ; \lambda)}\right|\left|\frac{\varphi_{\Pi}(m ;-\lambda, a)}{c_{\Pi}^{+}(m ;-\lambda)}\right| .
$$

By Lemma 4.1 (e), there are constants $C>0$ and $r \geq 0$ (depending on $m$ ) such that

$$
\left|c_{\Pi}^{+}(m ;-\lambda)\right|^{-1} \leq C(1+|\lambda|)^{r}
$$

for all $\lambda \in i \mathrm{a}^{*}$. Theorem 2.7(b) proves the existence of a constant $C^{\prime}$ (depending on $m$ ) such that

$$
\left|\varphi_{\Pi}(m ;-\lambda, a)\right| \leq C^{\prime}
$$

for all $\lambda \in i \mathfrak{a}^{*}$ and $a \in A_{\ominus}$. Finally, Corollary 4.3 yields for all $\lambda \in i \mathfrak{a}^{*}$ and all $n \in \mathrm{N}$

$$
\left|\frac{\mathscr{F}_{\Theta} f(m ; \lambda)}{c_{\Theta}(m ; \lambda)}\right| \leq C_{\Theta, N, m, f}(1+|\lambda|)^{-N} .
$$

By selecting $N \in \mathrm{N}$ large enough, the required convergence is achieved.

Remark 4.6. Since $\varphi_{\Pi}(m ; \lambda, a)=E_{\Pi}(m ; \lambda, a)$, the version of the inversion formula given by Theorem 4.5 is the exact analog for the set $\Theta \subset \Pi$ of Opdam's inversion formula for the transform associated with the hypergeometric functions associated with root systems. Up to a constant multiple, it agrees with Opdam's inversion formula for $\Theta=\Pi$, and hence with the inversion formula for the spherical transform on Riemannian symmetric spaces in the geometric case (see e.g. [2], Theorem 6.4.1). In the geometric case of noncompactly causal symmetric spaces, the inversion formula reduces to Ólafsson's formula (Theorem 6.13 in [10]. See also Corollary 4.8).

For future reference we record the inversion formula as deduced from Theorem 4.5 for the transform corresponding to Harish-Chandra series (i.e. when $\Theta=\emptyset)$ and for the spherical Laplace transform on NCC symmetric spaces.

COROLlaRY 4.7. There is a constant $k>0$ (depending only on the normalization of the measures) so that for all $f \in C_{c}^{\infty}\left(A^{+}\right)$the following inversion formula holds: For all $a \in A_{\Theta}$

$$
f(a)=k|W| \int_{i a^{*}} \mathscr{F}_{\emptyset} f(m ; \lambda) \varphi_{\Pi}(m ;-\lambda, a) \frac{d \lambda}{c_{\Pi}^{+}(m ;-\lambda) c_{\emptyset}^{-}(m ; \lambda)},
$$

where $d \lambda$ is a suitable normalization of the Lebesgue measure on $i a^{*}$.

Let $G / H$ be a NCC symmetric space. We assume the notation of Chapter 8 in [8]. In particular, we denote by $c_{\max }$ the maximal cone in $a$ associated with the causal structure. The $c_{\max }$ is invariant under the small Weyl group $W_{0}$ (see also 
Example 2.4). Let $c_{\max }^{0}$ be the interior of $c_{\max }$, and set $S^{0}:=H\left(\exp c_{\max }^{0}\right) H$. Let $\varphi_{\lambda}$ and $\psi_{\lambda}^{d}$ respectively denote the spherical functions on $G / H$ and on its Riemannian dual $G / K$. As common in this context, we write $\delta(a)$ instead of $\delta(m ; a)$.

Corollary 4.8 ([10], Theorem 6.13). Let $f$ be a $H$-bi-invariant $C^{\infty}$ function on $S^{0} / H$ such that $\left.f\right|_{\exp c_{\max }}$ is compactly supported. Then the spherical Laplace transform

$$
\mathscr{L} f(\lambda):=\int_{S^{0} / H} f(x) \varphi_{\lambda}(x) d x=\sigma \int_{A^{+}} f(a) \varphi_{\lambda}(a) \delta(a) d a
$$

is inverted by

$$
f(a)=\sigma^{\prime} \frac{|W|}{\left|W_{0}\right|} \int_{i a^{*}} \mathscr{L} f(\lambda) \psi_{-\lambda}^{d}(a) \frac{d \lambda}{c^{d}(-\lambda) c(\lambda)},
$$

where $c(\lambda)$ is the c-function on $G / H$ and $c^{d}(\lambda)$ is Harish-Chandra's c-function on $G / K$. Here $d \lambda$ is a suitable normalization of the Lebesgue measure on $i \mathfrak{a}^{*}$, and $\sigma$ and $\sigma^{\prime}$ are constants only depending on the normalization of the measures.

Proof. In the notation of [8] and Example 2.4, we have

$$
\begin{aligned}
c^{d}(\lambda) & =\frac{c_{\Pi}^{+}(m ; \lambda)}{c_{\Pi}^{+}(m ; \rho(m))}, & c(\lambda) & =\frac{c_{\Pi_{0}}(m ; \lambda)}{c_{\Pi_{0}}(m ; \rho(m))}, \\
\psi_{\lambda}^{d}(a) & =\frac{\varphi_{\Pi}(m ; \lambda, a)}{c_{\Pi}^{+}(m ; \rho(m))}, & \varphi_{\lambda}(a) & =\frac{\varphi_{\Pi_{0}}(m ; \lambda, a)}{c_{\Pi_{0}}(m ; \rho(m))} .
\end{aligned}
$$

\section{REFERENCES}

1. Faraut, J., Hilgert, J., and Ólafsson, G., Spherical functions on ordered symmetric spaces, Ann. Inst. Fourier (Grenoble) 44 (1994), no. 3, 927-965.

2. Gangolli, R., and Varadarajan, V. S., Harmonic Analysis of Spherical Functions on Real Reductive Groups, Springer-Verlag, Berlin, 1988.

3. Hawking, S. W., and Ellis, G. F. R., The Large Scale Structure of Space-time, Cambridge University Press, London-New York, 1973.

4. Heckman, G. J., Dunkl operators, Séminaire Bourbaki, Vol. 1996/97. Astérisque No. 245 (1997), Exp. No. 828, 4, 223-246.

5. Heckman, G. J., and Opdam, E. M., Root systems and hypergeometric functions. I, Compositio Math. 64 (1987), no. 3, 329-352.

6. Heckman, G., and Schlichtkrull, H., Harmonic Analysis and Special Functions on Symmetric Spaces, Academic Press, 1994.

7. Helgason, S., Groups and Geometric Analysis. Integral Geometry, Invariant Differential Operators, and Spherical Functions, Academic Press, 1984. 
8. Hilgert, J., and Ólafsson, G., Causal Symmetric Spaces. Geometry and Harmonic Analysis, Academic Press, 1996.

9. Krötz, B., and Ólafsson, G., The c-function for non-compactly causal symmetric spaces, Invent. Math. 149 (2002), no. 3, 647-659.

10. Ólafsson, G., Spherical functions and spherical Laplace transform on ordered symmetric spaces, Preprint, 1997. Available at http://www.math.lsu.edu//preprint.

11. Ólafsson, G., and Pasquale, A., On the meromorphic extension of the spherical functions on noncompactly causal symmetric spaces, J. Funct. Anal. 181 (2001), no. 2, 346-401.

12. Ólafsson, G., and Pasquale, A., Regularity properties of generalized Harish-Chandra expansions. In A. Strasburger et al., Geometry and analysis on finite- and infinite-dimensional Lie groups (Bedlewo, 2000), 335-348, Banach Center Publ., 55, Polish Acad. Sci., Warsaw, 2002.

13. Ólafsson, G., and Pasquale, A., Fourier-Laplace transforms associated with root systems. Geometry and harmonic analysis. In preparation.

14. Opdam, E. M., Root systems and hypergeometric functions. III, Compositio Math. 67 (1988), no. $1,21-49$.

15. Opdam, E. M., Root systems and hypergeometric functions. IV, Compositio Math. 67 (1988), no. 2, 191-209.

16. Opdam, E. M., Harmonic analysis for certain representations of graded Hecke algebras, Acta Math. 175 (1995), no. 1, 75-121.

17. Opdam, E. M., Lecture notes on Dunkl operators for real and complex reflection groups, MSJ Memoirs, 8. Mathematical Society of Japan, Tokyo, 2000.

18. Pasquale, A., A theory of $\Theta$-spherical functions, Habilitationsschrift, Technische Universität Clausthal, 2002.

19. Unterberger, J., Analyse harmonique sur un espace symétrique ordonné et sur son dual compact, $\mathrm{PhD}$ thesis, Université Pierre et Marie Curie, Paris, 1999.

INSTITUT FÜR MATHEMATIK

TU-CLAUSTHAL

38678 CLAUSTHAL-ZELLERFELD

GERMANY
PRESENT ADDRESS:

LABORATOIRE ET DÉPARTEMENT DE MATHÉMATIQUES, UNIVERSITÉ DE METZ, ILE DU SAULCY

F-57045 METZ

FRANCE

E-mail: pasquale@math.univ-metz.fr 